\title{
Petri Net Verification of Service Composition Mechanism based on Hierarchical Service Overlay Network
}

\author{
Yu Qiao, Yanmei Zhang, Hengyue Jia \\ Information School of Central University of Finance and Economics, Beijing 100081, China \\ jlzym0309@sina.com
}

Keyword: overlay network; service composition; service overlay network ; mechanism of composition ; Petri net

\begin{abstract}
This paper proposed a hierarchical service overlay network framework (HSON) based on the analysis of advantages and disadvantages of the present service composition network framework and the characteristics of service composition applications, and studied service composition mechanism based on HSON.. The Petri net is adopted to describe the service composition mechanism, and verify the correctness and completeness. In the end, we used examples to verify the reasonable of using HSON to get service compositon.
\end{abstract}

\section{Introduction}

Service composition can depend on user demands to put some discrete, existing fine granular basic services into complex corse-grained services with new functions, so as to realize the development and customization of the services. This is a mainstream of development direction of the network service in the future, and will be increasingly extensive applied. There still exist some problems in the current service composition technologies, especially the lack of unification of the research about the key technical problems under the appropriate network architecture, which makes the service composition limited in some specific application fields and the smaller range of network. But with the progress of Internet technology and the continuous development of Service Composition application, to provide users with Service composition which are various fields oriented, wan range and based on QoS guarantee is the future trend of service composition development, so this paper proposes a hierarchical service overlay network framework HSON which is service composition oriented. We also described and validated the Service composition mechanism based on HSON.

\section{Hierarchical service overlay network framework HSON}

Overlay network caused attention and received extensive development because of some characters of itself, such as high flexibility, scalability, easy to implement and deployment new services. Service overlay network frameworks relevant with services currently mainly include two categories.

First, it is the overlay network framework which builds on the communication subnet. Among this, service overlay network which represented by paper [1] is built overlay network on the communication subnet, and deploys services gateway between different ISP. Logical connection between two Service Gateway is provided by the bottom layer's ISP and it must satisfy the bandwidth request and service qualities, and the service qualities are defined by a bidirectional SLA. Service overlay network bypasses the connection point between ISP in this way in order to avoid the resultant performance problems and guaranteed the good performance of the end-to-end application which across multiple ISP. There are a lot of researches related to this thought, for example, Paper [2],[3] and [4] studied the problems in the construction process of the overlay network. Paper [5] and [6] studied the topology configuration problems of overlay network. This type of overlay network is mainly to solve the routing problems between different ISP in the service network, but it costs too much in construction. Meanwhile, it can not only deployed on a large 
number of services in the cover nodes. Therefore, it is not suitable for constructing the service composition network framework.

Second, it is P2P network framework. Paper [7] constructed a framework of service composition based on P2P network characteristics including no center, self-organizing and high efficient routing and so on. But considering the problems such as security, easy to manage in the process of service composition, service components generally distributed in the high performance controllable node in the network instead of distributed on the end-to-end system. So it will bring security problems and other issues by using P2P system as network framework of service composition.

After we deeply studied the advantages and disadvantages in the current service composition framework, we propose a service composition framework oriented to service composition which called hierarchical overlay network framework(HSON), as is shown in Fig1. Our basic idea is: first of all, use the autonomous domain as the basic units. Then, collect a large number of available content servers of the network,

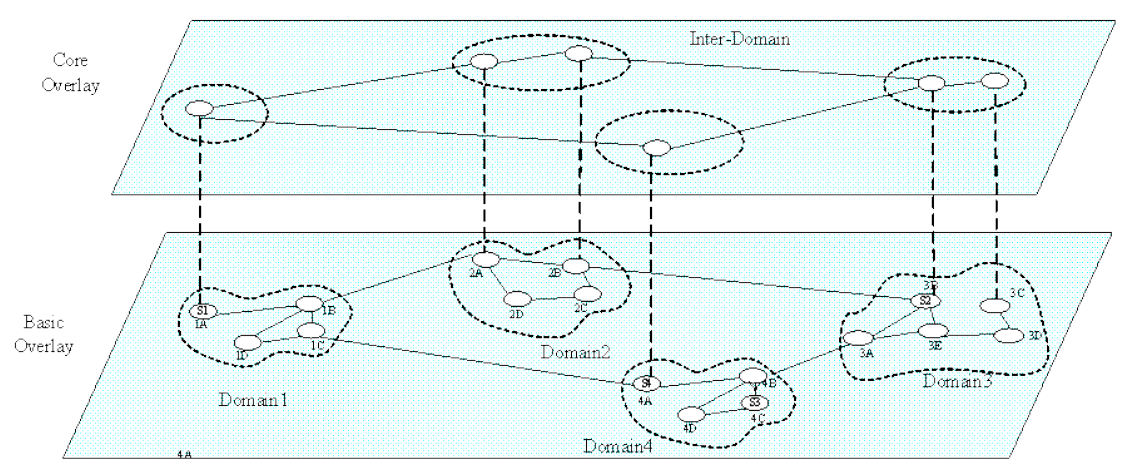

Fig.1 hierarchical service overlay network framework and construct domain overlay network by the method of deploying several proxy servers of service composition.Next, chose some super nodes in every domain depending on the needs of service composition in inter-domain, and built the network of inter-domains with the super nodes which called core overlay network as well. Finally, establish service index of inter-domain based on the correlation between the services and other factors. Through these three steps, hierarchical overlay network framework (HSON) was created successfully.

HSON not only has high efficiency of physical network, but also has the flexibility of overlay network, and can deploy a large number of service components according to the correlation between services, so it can provide users with applications of service composition which can be implemented in a WAN range, and many fields of application. An application of Service composition can occur in an autonomous domain, and we call these services as service composition in domain. An application of Service composition can also take place in many autonomous domains, and we call it service composition in inter-domains. According to the mode of service composition, the structure of service composition can be linear and parallel. This paper using HSON as service composition framework, to study the mechanism of service composition based on HSON. The verification of reasonableness of workflow Model has become an important part of the work flow system, and has played an important role in safe and stable operation of the entire system.

\section{Description of service composition mechanism based on HSON}

We use the examples of response procedure of user request to describe the service composition mechanism based on HSON. To better describe this, we ignored some treatment about anomalies problems such as the service server suddenly crashes when in a service process. We will introduce the procedure of service composition based on HSON Model followed.

(1) Users send service requests to local proxy servers of service composition.

(2) After receiving a request for content, local proxy servers of service composition will find the components which meet the demands of service composition in its own service directory. If all the components are found, local proxy servers of service composition will complete the work of choosing routing way of service composition: First, judge the routing structure of service composition, if it is a linear structure, then excute the algorithm of service routing choosing for linear structure to choose routing; while, if it is a parallel structure, then excute the algorithm of 
service routing choosing for parallel structure to choose routing. Finally, send response messages to the user.

If there exists no service component required to the service composition, or has found the demanding service components, but the load capacity of the node where the service components have already reached the limit, then forwarding service composition request to other proxy servers of service composition in domain.

(3) Other proxy servers of service composition will find the components for demand of service composition in its own service directory after receiving a request for content. If all the components are found, sending service components address list to local proxy servers of service composition, and local proxy servers of service composition will complete the work of choosing the routing way of service composition. Proxy servers of service composition in domains are responsible for service composition in domains to collaborative complete this work.

If there exists no service component which met the needs to the service composition, then forwarding request of service composition to super nodes in domains.

(4) Super nodes in domains will find the components which meet the demands of service composition in its own service directory of inter-domains. If all the components are found in other autonomous domains, then super nodes in domains will complete the work of choosing routing way in inter-domains of service composition. And send service response message to local proxy servers of service composition. Then local proxy servers of service composition send service response message to users. Super nodes in domains are responsible for service composition in inter-domains to collaborative complete this work.

Otherwise, forward request of service composition to super nodes in other domains.

(5) Find the components which meet the demands of service composition in the whole HSON. If all the components are found, then every super nodes in autonomous domains will complete the work of choosing routing way in inter-domains of service composition. For service composition in domains which possible exist, we excute the corresponding choosing algorithm of service routing in domains. And send service response message to local proxy servers of service composition. Then local proxy servers of service composition send service response message to users. Super nodes in domains are responsible for service composition in inter-domains to collaborative complete this work.

If there exists no service component which met the needs to the service composition, then send rejected service response message to users.

(6) User will get service composition and establish sessions with nodes in routing path of service composition or autonomous domains with the collaborative help of local proxy servers of service composition after they have received service response messages.

Give up this request when users received a rejected response message or waiting timeout after sent request.

\section{Petri nets Verification of the mechanism of service composition based on HSON}

According to the description of service composition mechanism based on the HSON in section 3, we can get the Petri net model as shown in Figure 2. Petri Net transition and the library are defined just as the shown in Table 1 and Table 2, each library's capacity is 1, each weight of the directed arcs is 1 . Since the super node and the details of the behavior of other proxy servers of the service compositions does not affect the whole mechanism about the local client sending a request, routing of service composition and response to users' request. So in order to simplify the Petri net's complexity, we do not describe the details of the behavior of these entities. 
Petri net shown in Figure 2 shows the case of the interaction between a local proxy server of service composition with a client. For each client and their local proxy servers of service composition, their working processes are identical and mutually independent, so if the running on a client and its local proxy server of service composition is correct, for the entire system, the agreement is also correct. In fact, in the case of multiple clients, the Petri net's description can be transformed to the petri net the same as figure 2 shows by Petri nets' folding technology, but library P0 and the initial capacity of the number of marks were changed to the number of the total customers' amount. On the contrary, the nature of the entire Petri net does not have any change. There exist conflicts of 8 pairs transitions of the Petri net shown in figure 2. T1 and T2 are triggered depending on the result of services' discovery in local proxy servers ' directory. T3 and $\mathrm{T} 4$ are triggered depending on the result of services' discovery in domains.T6 and T7 are triggered depending on the result of services'

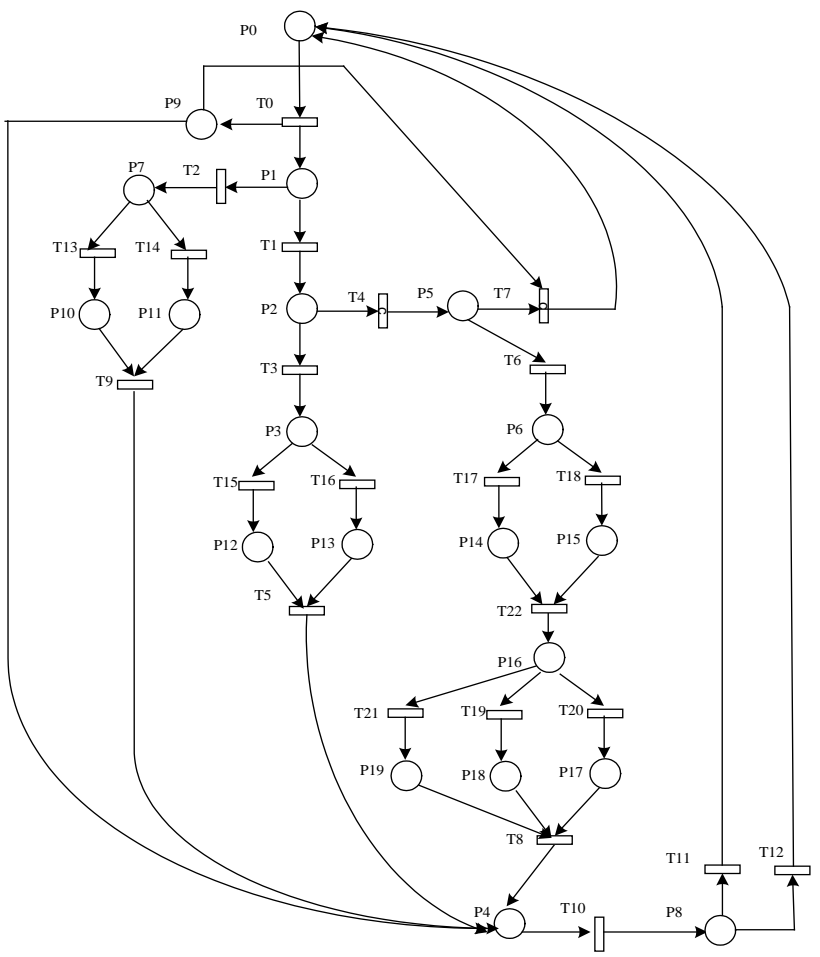

Fig.2 Petri description of service composition based on HSON discovery in inter-domains. T11 and T12 are triggered depending on whether the route is faulty during the process of service composition. T13 and T14, T15 and T16 are triggered depending on the structure of the routing path of service composition.T19, T20 and T21 are triggered depending on the structure of the routing path of service composition, as well as whether service composition in inter-domains including the service composition in domains.

Transition diagram of Libraries can directly show the interaction process of entities in service composition mechanism, at the same time, the complex asynchronous interaction of multiple entities may have unpredictable consequences. Therefore, this paper verified the correctness and completeness of service composition mechanism based on HSON m using the analysis method of the Petri net.

We can prove the correctness and completeness of the Petri net system by the method of reachability graph. We build the reachability graph of system based on enumeration method of reachability graph to showing the identities of each network and occurrence of every single transition. If the network system is bounded, then the reachability graph is limited, and we can prove its properties easily. Just as the figure 3 shows, we build the reachability graph of Petri net system as shown in figure 2 using the method of reachability graph. We can prove the correctness and completeness of the Petri net system theoretically with reachability graph. 
Table1:Definition of library of Petri net

\begin{tabular}{|c|l|}
\hline Number & \multicolumn{1}{|c|}{ Definition } \\
\hline P0 & the client Prepare to send a request \\
\hline P1 & $\begin{array}{l}\text { local proxy server of service composition receives a request, then find service } \\
\text { which are needed }\end{array}$ \\
\hline P2 & find service which are needed and forwarding service requests in the domain \\
\hline P3 & Judge and abstract the routing path structures of service composition in domain \\
\hline P4 & Client obtained routing information of service composition \\
\hline P5 & Send a request for global search in the core overlay network \\
\hline P6 & $\begin{array}{l}\text { Judge and abstract the routing path structures of service composition in } \\
\text { inter-domain }\end{array}$ \\
\hline P7 & Judge and abstract the routing path structures of service composition in domain \\
\hline P5 & Clients receive services \\
\hline P9 & The client waits for a reply message \\
\hline P10 & $\begin{array}{l}\text { local proxy server excute the algorithm of service routing choosing in domains } \\
\text { for linear structure }\end{array}$ \\
\hline P11 & $\begin{array}{l}\text { local proxy server excute the algorithm of service routing choosing in domains } \\
\text { for parallel structure }\end{array}$ \\
\hline P12 & $\begin{array}{l}\text { proxy servers of service composition are responsible for collaborative excute the } \\
\text { algorithm of service routing choosing in domains for linear structure }\end{array}$ \\
\hline P13 & $\begin{array}{l}\text { proxy servers of service composition are responsible for collaborative excute the } \\
\text { algorithm of service routing choosing in domains for parallel structure }\end{array}$ \\
\hline P14 & $\begin{array}{l}\text { proxy servers of service composition are responsible for collaborative excute the } \\
\text { algorithm of service routing choosing in inter-domains for linear structure }\end{array}$ \\
\hline P15 & $\begin{array}{l}\text { proxy servers of service composition are responsible for collaborative excute the } \\
\text { algorithm of service routing choosing in inter-domains for parallel structure }\end{array}$ \\
\hline P16 & Judge whether contain the intra-domain routing and the structures of routing \\
\hline P17 & $\begin{array}{l}\text { Super nodes are responsible for collaborative excute the algorithm of service } \\
\text { routing choosing in domains for parallel structure }\end{array}$ \\
\hline P18 & $\begin{array}{l}\text { Super nodes are responsible for collaborative excute the algorithm of service } \\
\text { routing choosing in domains for linear structure }\end{array}$ \\
\hline P19 & \begin{tabular}{l} 
no physical meaning \\
\hline
\end{tabular} \\
\hline
\end{tabular}

Table2:Definition of transitions of Petri net

\begin{tabular}{|c|l|}
\hline Number & \multicolumn{1}{|c|}{ Definition } \\
\hline T0 & $\begin{array}{l}\text { Client sent request of service composition to local proxy server of service } \\
\text { composition }\end{array}$ \\
\hline T1 & $\begin{array}{l}\text { services we need are not found or did not all found in local proxy server } \\
\text { directory }\end{array}$ \\
\hline T2 & find all the services in local proxy server directory \\
\hline T3 & find all the services in the in domain \\
\hline T4 & services we need are not found or did not all found in the in domain \\
\hline T5 & Sent routing information in domain to the client \\
\hline T6 & Find all the services in the core coverage network \\
\hline T7 & $\begin{array}{l}\text { If services we need are not found or did not all found in the core network, then } \\
\text { fails }\end{array}$ \\
\hline T8 & Sent inter-domain routing information to the client \\
\hline T9 & sent routing information in domain to the client \\
\hline T10 & Start process of service composition \\
\hline T11 & successful implementation of service composition \\
\hline T12 & routing failure, service failure \\
\hline T13 & Linear structure in domain \\
\hline T14 & parallel architecture in domain \\
\hline T15 & linear structure with domain \\
\hline T16 & parallel architecture in domain \\
\hline T17 & inter-domain linear structures \\
\hline T18 & inter-domain parallel structure \\
\hline T19 & inter-domain routing domain includes linear structures in domain \\
\hline T20 & inter-domain routing includes parallel structures in domain \\
\hline T21 & inter-domain routing does not contain routing in domain \\
\hline T22 & no physical meaning \\
\hline &
\end{tabular}

The states underlined in figure 3 coincide with the initial state and form a loop, for clarity, we do not draw any direct connection with the arc. Customer request-response protocol to run properly for the following situations: It is not difficult to see that all the sequence of transition above shown in the diagram shown in Figure 3, and each of them is located in different basic circles which under the node of initial state, this can indicate that these sequence of transition can be repeated unlimited. We can also find that all possible states from the initial state are reachable and are at different basic circles from the figure 3 . Therefore, it can not be a deadlock, and it will not appear endless loop, either. So a variety logic behaviors of systems are correct. All the number of Token in each library are no more than one, and the Petri net is bounded, thus it can ensure the low occupancy rate of system resources. For the case of multiple clients, since the provisions of customers can only accept a service composition at one time in max which means that the number of initial token of he capacity of libraries P0 are limited to 1 , as long as the number of customers is limited, due to the heavyweight of each arc is 1 , the maximum number of each library token will not exceed the number of customers.So the system is bounded. All sequences of transition will happen according to the above analysis, and the bounded of Petri nets ensures

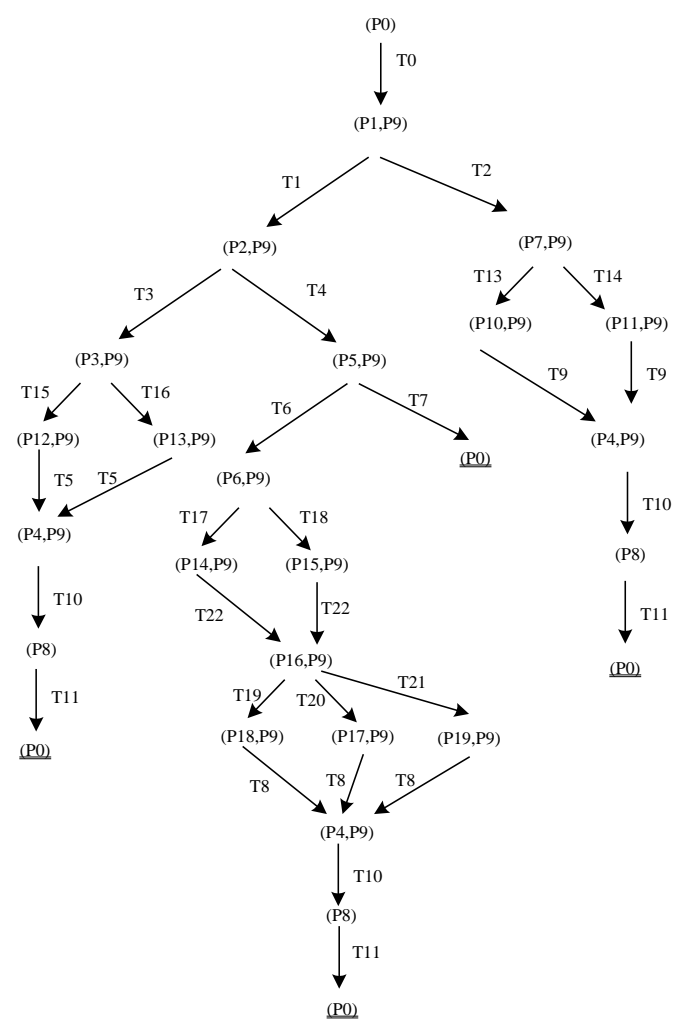

Fig.3 reachability graph of Service composition procedure based on HSON that the consumption of resources is limited, so the system is correct. But we can also be seen from the diagram, the system can return to the initial state no matter what state the system is on, and all its transitions can be triggered in no limited times, so the system will not fall into one of unlimited local circulation, no "hunger" phenomenon will happen, and the deadlock or the live lock will not occur, either.At the same time, we can say that the reachable states of system are reasonable and effective, and the network system is safe, bounded. Therefore, we can prove its completeness. In 
summary, all the states of the mechanisms of service composition are reachable, no deadlocks and live locks, and the mechanism of service composition is correct and completed.

\section{Conclusion}

We proposed an service-oriented hierarchical service overlay Network(HSON) and studied the service composition mechanism based on HSON according to the advantages in supporting new network applications and the characteristics of service composition applications. And we described the service composition mechanism based on HSON and verified its correctness. We will further studied the problems about the service discovery and service routing algorithm which based on HSON in the future.

\section{Acknowledgments}

This study was supported by the National Natural Science Foundation of China (No.61309029, 61273293) and key projects of social science fund of Beijing city (No. 16YJA001) and Discipline Construction Foundation of Central University of Finance and Economics.

\section{References}

[1] Zhenhai Duan.ZhiLi Zhang.Yiwei Thomas Hou Service overlay networks:SLAs,QoS,and bandwidth provisioning[J], IEEE/ACM Transactions on Networking (TON),Volume 11 , Issue 6 (December 2003):: 870 - 883 ,2003

[2]Jin Xiao, Raouf Boutaba:Reconciling the overlay and underlay tussle,IEEE/ACM Transactions on Networking (TON): Volume 22 Issue 5, October 2014

[3]Pawel Garbacki, Dick H. J. Epema, Maarten van Steen: Broker-placement in latency-aware peer-to-peer networks[J]. Computer Networks 52(8): 1617-1633 (2008)

[4] Kyuho Jeong, Renato Figueiredo:Self-configuring Software-defined Overlay Bypass for Seamless Inter- and Intra-cloud Virtual Networking

Proceedings of the 25th ACM International Symposium on High-Performance Parallel and Distributed Computing,2016

[5]Young Yoon, Nathan Robinson, Vinod Muthusamy, Sheila McIlraith, Hans-Arno Jacobsen:Planning the transformation of overlays.Proceedings of the 31st Annual ACM Symposium on Applied Computing.2016

[6]Jaesung Park, Yujin Lim:An overlay network construction method using swarm intelligence for an application-level multicast service.Proceedings of the 2013 Research in Adaptive and Convergent Systems.2013

[7]Manel Seddiki, Mahfoud Benchaïba:An adaptive P2P overlay for MANETs.Proceedings of the 2015 International Conference on Distributed Computing and Networking.2015

\section{Authors}

Qiao Yu (1996-), female, is currently an undergraduate at the Information School of CUFE(Central University of Finance and Economics), She is a leader of a national innovation training program, and won the National Endeavor Fellowship, academic scholarship of undergraduates, scholarships of all-round development and so on. Her interest of research areas including service computing, granular computing.

Yanmei Zhang received her Ph.D. degree in 2010 from the China University of Mining and Technology (CUMT) in Beijing, China. She is an Associate Professor of the Department of Computer Science and Technology at CUFE. Her general areas of research are Intelligent data 
analysis, and service computing. She has published more than 40 papers in refereed conferences and journals. Her research is supported by the National Natural Science Foundation of China..

Hengyue Jia received her Ph.D. degree in 2012 from the Beijing University of Posts and Telecommunications (BUPT) in Beijing, China. She is a lecturer of the Department of Information Security at CUFE. Her general areas of research are Intelligent data analysis, secure multiparty computation and quantum information processing. She has published more than 10 papers in refereed conferences and journals. 